\title{
Checklist da ictiofauna do Estado de Mato Grosso do Sul, Brasil
}

\author{
Otávio Froehlich', Marcel Cavallaro², José Sabino³, Yzel Rondon Súarez ${ }^{4}$ \\ \& Maria José Alencar Vilela ${ }^{5}$
}

\begin{abstract}
1. Universidade Federal de Mato Grosso do Sul, Laboratório de Zoologia/CCBS. Campo Grande, MS, Brasil. In memoriam.
2. Universidade do Estado da Bahia, Departamento de Educação, Campus VIII. Rua do Bom Conselho, 179, Alves de Souza, 48608-230 Paulo Afonso, BA, Brasil.

3. Universidade para o Desenvolvimento do Estado e da Região do Pantanal, Pró-Reitoria de Pesquisa e Pós-Graduação. Rua Alexandre Herculano, 1400

Campus III, Jardim Veraneio, 79037-280 Campo Grande, MS, Brasil. (sabino-jose@uol.com.br)

4. Universidade Estadual de Mato Grosso do Sul, Centro Integrado de Análise e Monitoramento Ambiental. Rod. Dourados-Itahum, Km 12, Cidade Universitária 79804-970 Dourados, MS, Brasil.

5. Universidade Federal de Mato Grosso do Sul/Campus Três Lagoas, Departamento de Ciências Naturais, Laboratório de lctiologia, Caixa Postal 210
\end{abstract}

Av. Ranulpho Marques Leal, 3484, Distrito Industrial, 79600-030 Três Lagoas, MS, Brasil.

Recebido 22 novembro 2016

Aceito 6 fevereiro 2017

DOI: $10.1590 / 1678-4766 e 2017151$

\begin{abstract}
Checklist of the Ichthyofauna from Mato Grosso do Sul state, Brazil. The freshwater fishes of Mato Grosso do Sul state are distributed in two Prata basin sub-basins, Paraguay and Upper Paraná. The species list for that state was compiled based on Collection records, literature and consultation with other ichthyologists. The list includes 358 species, belonging to eleven orders and 43 families. For the Paraguay basin, 255 species were listed; 201 were registered in the Upper Paraná basin. At least 100 species are present in the two watersheds. Of those, 49 species are indigenous to the two basins. Among the other shared species, three were introduced into both basins and 45 are native to the Paraguay and/or Middle Parana basins and were intentionally or inadvertently introduced into the Upper Paraná basin. Nearly one third of the species registered in the Upper Parana basin (64), in Mato Grosso do Sul were introduced, whereas introductions in the Paraguay basin correspond to $1.2 \%$ of the species listed. The number of species presented in this paper is considered to be low, representing the still incomplete knowledge, specially of the headwater regions, but also of the lowlands of the Pantanal. Threats to this ichthyofauna and aspects of its conservation status are also discussed.
\end{abstract}

KEYWORDS. Paraguay River basin, upper Parana River basin, fish, species list, Biota-MS Program.

RESUMO. Os peixes de água doce do estado de Mato Grosso do Sul se distribuem em duas sub-bacias da bacia do Prata, Paraguai e Alto Paraná. A lista das espécies de peixes que ocorrem no estado de Mato Grosso do Sul foi compilada com base em dados de coleções, literatura e consulta a especialistas. São registradas 358 espécies, distribuídas por onze ordens e 43 famílias. Para a bacia do Paraguai são listadas 255 espécies; 201 o foram para a bacia do Alto Paraná. Cem espécies foram listadas para as duas bacias. Destas, 49 são espécies que ocorrem naturalmente nas duas bacias. Das outras espécies compartilhadas, três foram introduzidas nas duas vertentes e 45 introduzidas na bacia do Alto Paraná, a partir das bacias do Paraguai ou Médio Paraná. Quase um terço das espécies arroladas para a bacia do Alto Paraná (64), em Mato Grosso do Sul, foram introduzidas; introduções na vertente do rio Paraguai representam $1,2 \%$ da lista para a bacia. O número de espécies é considerado ainda baixo, representando um conhecimento ainda bastante incompleto, especialmente das regiões de cabeceiras do estado, mas também das terras baixas do Pantanal. Aspectos relacionados à conservação da ictiofauna do estado também são abordados.

PALAVRAS-CHAVE. Bacia do Paraguai, bacia do Alto Paraná, peixes, lista de espécies, Programa Biota-MS.

O estado de Mato Grosso do Sul é drenado por duas subunidades da bacia do Prata - as bacias do Alto Paraná, a leste, e do Paraguai, a oeste. O divisor de águas entre as duas sub-bacias, de sudoeste para nordeste, divide o estado em duas áreas de tamanhos semelhantes. A porção drenada diretamente pelo rio Paraná representa 47,5\% da área do estado $\left(169.488,66 \mathrm{~km}^{2}\right)$, enquanto que a vertente do rio Paraguai (52,5\% da área do estado) tem $187.636,30 \mathrm{~km}^{2}$, segundo dados disponíveis no ZonEAMENTo ECOLÓGICOEcONÔMico do Estado de Mato Grosso do Sul (ZEE-MS) (http://www.semac.ms.gov.br/zeems/).

NeLSON (2006) estimou a existência de 28.000 espécies de peixes no mundo. ReIs et al. (2003) arrolaram 4.475 espécies em águas continentais da Região Neotropical e citaram 1.550 formas reconhecidamente novas mas ainda não descritas e BUCKUP et al. (2007) compilaram 2.587 espécies para as águas doces brasileiras. Portanto, não seria exagero dizer que o Brasil abriga cerca de $10 \%$ das espécies de peixes do mundo.

Caracterização da Área. Praticamente metade (ca. $90.000 \mathrm{~km}^{2}$ ) da área banhada pela bacia do Paraguai é ocupada, no estado, pelo complexo bioma do Pantanal, planície sazonalmente inundável pelas águas do rio Paraguai e seus principais afluentes. A leste do Pantanal, na Região dos Planaltos (ZEE-MS), com seus três Patamares (ZEE$\mathrm{MS})$, encontra-se a maior parte das cabeceiras do rios que descem para o Pantanal. Na região sudoeste de MS, entre os Planaltos e a planície inundável (aqui estreita) está o Planalto 


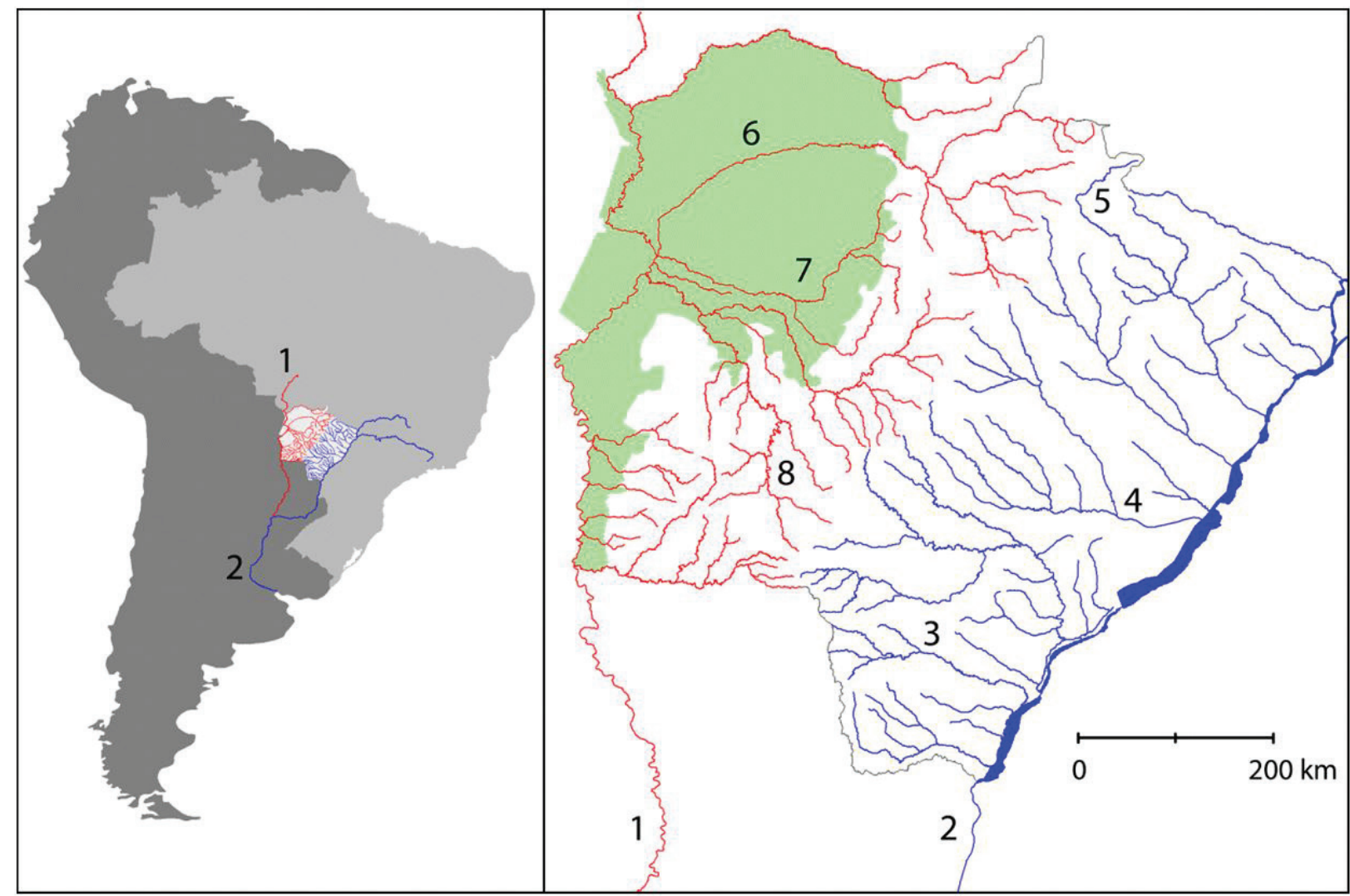

Fig.1. Hidrografia do estado de Mato Grosso do Sul e sua posição na América do Sul (esquerda), com destaque para os seus principais formadores (direita) (azul, bacia do Alto rio Paraná; vermelho, bacia do rio Paraguai; verde, Pantanal; 1, rio Paraguai; 2, rio Paraná; 3, rio Amambai; 4, rio Pardo; 5 , rio Sucuriú; 6, rio Taquari; 7 , rio Negro; 8 , rio Miranda.

da Bodoquena, um afloramento de rochas carbonáticas. Nesta região estão as cabeceiras da margem esquerda do rio Miranda e parte do rio Apa.

A parte do Mato Grosso do Sul drenada pelo rio Paraná é mais uniforme. Seus rios cortam as formações areníticas dos grupos Botucatu e Bauru do planalto sedimentar do Paraná, com relevo de ondulação suave e altitudes que decrescem paulatinamente de noroeste para sudeste.

As regiões mais altas do estado são os Planaltos Residuais do Paraná (ZEE-MS), na porção centro-norte, com áreas relativamente planas entre 800 e 900 metros. O Planalto da Bodoquena e a borda ocidental do planalto sedimentar do Paraná apresentam altitudes entre 500 e 600 metros. No maciço do Urucum, próximo a Corumbá, e na Serra do Amolar (extremo NO), picos beiram os 1.000 metros. O longo dos rios Paraná e Paraguai as altitudes médias são de 280 e 85 metros, respectivamente.

\section{MATERIAL E MÉTODOS}

Para a confecção da lista de espécies de peixes foram utilizadas informações de diferentes fontes. Uma primeira listagem foi obtida pela busca de lotes procedentes de Mato Grosso do Sul tombados naquelas coleções que têm seu acervo já informatizado e disponível para consulta pública (dentre elas DZSJRP-Pisces, INPA-Peixes, LIRP, MCPPeixes, MHNCI-Peixes, MZUEL-Peixes, MZUSP, UFRGS,
ZUEC-PIS), via speciesLink (http://splink.cria.org.br/).

Adições a essa lista foram feitas por consulta direta a acervo de outras coleções, via rede (CAS, FMNH, NUP, USNM), ou in loco (ZUFMS-PIS) e por meio de literatura, envolvendo catálogos (REIs et al., 2003; BUCKUP et al., 2007; FERRARIS JR., 2007), revisões regionais (BRITSKI et al., 2007; Graça \& Pavanelli, 2007), checklists (Langeani et al., 2007; OYAKAWA \& MENEZES, 2011) e artigos que envolvem descrição de novas espécies e/ou revisões de grupos. Além disso, algumas poucas espécies foram incluídas após consulta a lotes não tombados disponíveis nos laboratórios dos autores e a outros especialistas. Estes últimos foram consultados também para a validação de alguns registros, o que rendeu a eliminação de algumas das espécies. Novas espécies, ainda não descritas formalmente, ou em processo de descrição, às quais não foi atribuído um nome, mesmo que incorreto, foram omitidas. No entanto, alguns nomes, hoje se sabe, representam complexos de espécies, foram mantidos na lista, sendo tratados como tal na discussão. Por fim, táxons cavernícolas não foram incluídos nesta listagem; essas espécies são apresentadas em CoRDEIRO et al. (2014).

A elaboração de uma lista de espécies de peixes de uma unidade geopolítica que abriga partes de bacias hidrográficas maiores é tarefa complicada (OYAKAWA \& Menezes, 2011). Para diminuir o risco de incluir espécies que, na verdade, não ocorrem no Mato Grosso do Sul, apenas espécies comprovadamente coletadas no estado foram 
incluídas na lista. Com isso, certamente foram omitidas espécies que ocorrem no Mato Grosso do Sul, mas não foram ainda coletadas nele, ou foram-no mas seus lotes não estão depositados em alguma das coleções pesquisadas.

\section{RESULTADOS E DISCUSSÃO}

São registradas 356 espécies de peixes para o estado de Mato Grosso do Sul (Tab. I). Duas espécies cavernícolas, Ancistrus formoso e Trichomycterus dali, não constam da lista; estão arroladas em CORDEIRo et al. (2014), elevando o total para 358 espécies. Essas espécies pertencem a 11 ordens, com a predominância usual de Characiformes e Siluriformes, e 43 famílias, sendo Characidae e Loricariidae aquelas com maiores números de espécies. Este número pode ser julgado baixo se comparado às 391 espécies do estado de São Paulo (Oyakawa \& Menezes, 2011), que tem $70 \%$ da área de Mato Grosso do Sul. É preciso considerar que São Paulo é o estado brasileiro cuja biodiversidade é a mais bem conhecida no país e contém drenagens atlânticas com alto grau de endemismo (ver OyaKaWA \& Menezes, 2011), além da drenagem do Alto Paraná que ocupa a maior parte do estado.

No Mato Grosso do Sul registramos 201 espécies para a bacia do Alto Paraná. A estimativa mais recente para o número de espécies que ocorrem nessa bacia cita 368 formas - 317 espécies descritas e 51 ainda não descritas formalmente (LANGEANi et al., 2007). Para a parcela da bacia contida no estado de São Paulo, Oyakawa \& Menezes (2011) listaram 260 espécies. A área da bacia no estado de São Paulo é cerca de $25 \%$ maior que aquela drenada pelo Alto Paraná em Mato Grosso do Sul, mas a principal razão para a menor riqueza de espécies no Mato Grosso do Sul ainda deve ser o menor grau de conhecimento, especialmente das regiões de cabeceiras no estado. Os cursos d'água de pequeno porte que drenam as áreas de cabeceiras dos rios maiores, são locais com grande potencial para a ocorrência de endemismos. A maior parte das espécies que habitam esses ambientes são de pequeno porte $(100-150 \mathrm{~mm})$, geralmente muito dependentes das condições ambientais encontradas nos riachos e apresentam baixa capacidade de deslocamento (CASTro \& Menezes, 1998). Com efeito, OyaKawa \& Menezes (2011) acreditam que 70 a $80 \%$ das espécies que arrolaram para São Paulo são habitantes de riachos.

Para a área de Mato Grosso do Sul drenada pelo rio Paraguai (Fig. 1) são listadas 257 espécies (255 na Tabela I mais as duas espécies troglóbias citadas anteriormente). O melhor termo de comparação disponível é BRITSKI et al. (2007), um extenso manual de identificação dos peixes que ocorrem no Pantanal e terras altas ao seu redor, no qual os autores apresentam 269 espécies. Poderia parecer que a Tabela I apresenta uma boa estimativa do número de espécies para a bacia do Paraguai no Mato Grosso do Sul, mas ela registra apenas 221 das espécies apresentadas em BRITSKI et al. (2007). Possivelmente, muitas das espécies citadas em BRITSKI et al. (2007) e não arroladas aqui ainda serão registradas em Mato Grosso do Sul. As espécies da
Tabela I que não constam daquela revisão são, em sua grande maioria, restritas às regiões de cabeceiras (que não eram o foco do trabalho) ou descritas depois ou pouco antes da publicação do livro.

De todas as espécies do MS, doze não são de ocorrência natural no estado, tendo sido introduzidas como consequência de ações humanas (Graça \& Pavanelli, 2007; LANGEANI et al. 2007; JÚLIO JR. et al., 2009 foram as principais fontes para a determinação de quais espécies foram introduzidas no estado e em cada bacia). Cinco delas são espécies exóticas para a ictiofauna brasileira. Tilapia rendalli foi trazida para o Brasil na década de 1950, para povoamento de reservatórios da CESP (Castagnoli, 1992). Seu uso muito frequente em pesqueiros e pisciculturas (GraÇA \& PaVANELli, 2007), permitiu larga disseminação pelo país. Em Mato Grosso do Sul, em ambientes naturais, $T$. rendalli tem sido registrada em alguns locais da bacia do Alto Paraná (FroeHLich et al., 2006; SÚAREZ et al., 2011 e dados das coleções). Na bacia do Paraguai, registros em coleções existem apenas para a parte alta do rio Perdido, no Planalto da Bodoquena (LIRP, ZUFMS-PIS). Oreochromis niloticus é outro ciclídeo africano trazido para o Brasil, este na década de 1970 (MoreIrA et al., 2007), também bastante utilizado em pesqueiros e pisciculturas (GraÇa \& PAVANELli, 2007) e, no estado, foi registrado em ambientes naturais da bacia do Alto Paraná. Poecilia reticulata, nativa da região do Caribe (Venezuela, Guianas e algumas ilhas; LUCINDA, 2003), foi extensivamente introduzida no Brasil e em boa parte das regiões tropicais e subtropicais do mundo (revisão em DEACON, 2010). Em alguns casos a introdução visou o controle de larvas de pernilongos (CHANDRA et al., 2008), mas a espécie é muito apreciada por aquaristas e soltura intencional ou acidental de espécimes também foi meio importante de disseminação (DEACON, 2010). Duas espécies de bagres exóticos também usadas em pisciculturas têm aparecido em ambientes naturais, de maneira esporádica: Clarias gariepinus, da África e Ásia Menor, e Ictalurus punctatus, da América do Norte. Estas duas espécies foram coletadas em Mato Grosso do Sul em águas da bacia do Alto Paraná, mas de forma episódica; nenhuma delas pode, ainda, ser considerada instalada na bacia, com registros representando escapes ocasionais (Zanatta et al., 2010; C. S. Pavanelli, com. pess.). Seis espécies amazônicas foram introduzidas em Mato Grosso do Sul. Três são ciclídeos - Geophagus cf. proximus (presente na bacia do Alto Paraná), provavelmente devido ao uso como espécie ornamental (GRAÇA \& PAVANELLI, 2007; LANGEANI et al., 2007) e duas espécies de tucunarés, Cichla kelberi (Alto Paraná) e $C$. piquiti (nas duas vertentes). Essas espécies foram introduzidas em vários reservatórios da bacia do Alto Paraná (ESPINOLA, 2009) visando a exploração via pesca esportiva e comercial. A segunda espécie também foi introduzida na bacia do Paraguai, mas de forma acidental. Um plantel era mantido em um viveiro de piscicultura, próximo à divisa MT-MS, que rompeu-se em 1982, introduzindo a espécie nos rios Itiquira e Piquiri (Marques \& Resende, 2005). Os dados desses autores sobre a distribuição indicam que já se dispersou para o sul, explorando águas mais limpas ao longo da margem 
Tab. I. Ictiofauna do estado de Mato Grosso do Sul, Brasil. (*, espécies ameaçadas; sobrescritos após indicativos de presença nas bacias indicam espécies introduzidas: 1 , introduzida via Itaipu ou canal da piracema; 2 , introduzida via piscicultura, por acidente ou proposital; 3 , introduzida via atividade pesqueira, devido a uso como isca viva; 4 , introduzida para controle de pernilongos; 5 , introduzida devido a uso como espécie ornamental; 6 , introdução de origem desconhecida; 7, dúvida sobre se a espécie é introduzida ou autóctone).

\begin{tabular}{|c|c|c|}
\hline & Alto Paraná & Paraguai \\
\hline \multicolumn{3}{|l|}{ Myliobatiformes } \\
\hline \multicolumn{3}{|l|}{ Potamotrygonidae } \\
\hline Potamotrygon falkneri Castex \& Maciel, 1963 & +1 & + \\
\hline Potamotrygon motoro (Müller \& Henle, 1841) & +1 & + \\
\hline \multicolumn{3}{|l|}{ Clupeiformes } \\
\hline \multicolumn{3}{|l|}{ Engraulidae } \\
\hline Lycengraulis grossidens (Spix \& Agassiz, 1829) & & + \\
\hline Pellona flavipinnis (Valenciennes, 1837) & & + \\
\hline \multicolumn{3}{|l|}{ Characiformes } \\
\hline \multicolumn{3}{|l|}{ Acestrorhynchidae } \\
\hline Acestrorhynchus lacustris (Lütken, 1875) & + & \\
\hline Acestrorhynchus pantaneiro Menezes, 1992 & & + \\
\hline \multicolumn{3}{|l|}{ Anostomidae } \\
\hline Abramites hypselonotus (Günther, 1868) & & + \\
\hline Leporellus vittatus (Valenciennes, 1850) & + & + \\
\hline Leporinus amblyrhynchus Garavello \& Britski, 1987 & + & \\
\hline Leporinus elongatus Valenciennes, 1850 & + & + \\
\hline Leporinus friderici (Bloch, 1794) & + & + \\
\hline Leporinus lacustris Amaral Campos, 1945 & + & + \\
\hline Leporinus macrocephalus Garavello \& Britski, 1988 & +2 & + \\
\hline Leporinus obtusidens (Valenciennes, 1837) & + & + \\
\hline Leporinus octofasciatus Steindachner, 1915 & + & \\
\hline Leporinus octomaculatus Britski \& Garavello, 1993 & & + \\
\hline Leporinus paranensis Garavello \& Britski, 1987 & + & \\
\hline Leporinus striatus Kner, 1858 & + & + \\
\hline Leporinus tigrinus Borodin, 1929 & +6 & \\
\hline Schizodon altoparanae Garavello \& Britski, 1990 & + & \\
\hline Schizodon borellii (Boulenger, 1900) & $+1,2$ & + \\
\hline Schizodon intermedius Garavello \& Britski, 1990 & + & \\
\hline Schizodon isognathus Kner, 1858 & & + \\
\hline Schizodon nasutus Kner, 1858 & + & \\
\hline \multicolumn{3}{|l|}{ Characidae } \\
\hline Aphyocharax anisitsi Eigenmann \& Kennedy, 1903 & +7 & + \\
\hline Aphyocharax dentatus Eigenmann \& Kennedy, 1903 & + & + \\
\hline Aphyocharax nattereri (Steindachner, 1882) & & + \\
\hline Aphyocharax rathbuni Eigenmann, 1907 & & + \\
\hline Aphyocheirodon hemigrammus Eigenmann, 1915 & + & \\
\hline Astyanax abramis (Jenyns, 1842) & & + \\
\hline Astyanax altiparanae Garutti \& Britski, 2000 & + & \\
\hline Astyanax asuncionensis Géry, 1972 & & + \\
\hline Astyanax biotae Castro \& Vari, 2004 & + & \\
\hline Astyanax bockmanni Vari \& Castro, 2007 & + & \\
\hline Astyanax fasciatus (Cuvier, 1819) & + & \\
\hline Astyanax lineatus (Perugia, 1891) & & + \\
\hline Astyanax marionae Eigenmann, 1911 & & + \\
\hline Astyanax paranae Eigenmann, 1914 & + & \\
\hline Astyanax pellegrini Eigenmann, 1907 & & + \\
\hline Astyanax schubarti Britski, 1964 & + & \\
\hline Brachychalcinus retrospina Boulenger, 1892 & & + \\
\hline Brycon hilarii (Valenciennes, 1850) & +2 & + \\
\hline Brycon orbygnianus (Valenciennes, 1850$)^{*}$ & + & \\
\hline Bryconamericus exodon Eigenmann, 1907 & +1 & + \\
\hline Bryconamericus iheringii (Boulenger, 1887) & + & \\
\hline Bryconamericus stramineus Eigenmann, 1908 & + & + \\
\hline Bryconamericus turiuba Langeani Lucena et al., 2005 & + & \\
\hline Bryconops melanurus (Bloch, 1794) & & + \\
\hline Catoprion mento (Cuvier, 1819) & & + \\
\hline Charax leticiae Lucena, 1987 & & + \\
\hline Clupeacharax anchoveoides Pearson, 1924 & & + \\
\hline Creagrutus meridionalis Vari \& Harold, 2001 & & + \\
\hline Creagrutus paraguayensis Mahnert \& Géry, 1988 & & + \\
\hline Creagrutus varii Ribeiro et al., 2004 & + & \\
\hline Ctenobrycon alleni (Eigenmann \& McAtee, 1907) & & + \\
\hline
\end{tabular}


Tab. I. Cont.

Cynopotamus argenteus (Valenciennes, 1836)

Cynopotamus kincaidi (Schultz, 1950)

Engraulisoma taeniatum Castro, 1981

Galeocharax humeralis (Valenciennes, 1834)

Galeocharax knerii (Steindachner, 1879)

Gymnocorymbus ternetzi (Boulenger, 1895)

Hemigrammus lunatus Durbin, 1918

Hemigrammus marginatus Ellis, 1911

Hemigrammus parana Marinho et al., 2008

Hemigrammus ulreyi (Boulenger, 1895)

Hyphessobrycon anisitsi (Eigenmann, 1907)

Hyphessobrycon elachys Weitzman, 1984

Hyphessobrycon eques (Steindachner, 1882)

Hyphessobrycon herbertaxelrodi Géry, 1961

Hyphessobrycon luetkenii (Boulenger, 1887)

Hyphessobrycon megalopterus (Eigenmann, 1915)

Hyphessobrycon moniliger Moreira et al., 2002

Jupiaba acanthogaster (Eigenmann, 1911)

Knodus moenkhausii (Eigenmann \& Kennedy, 1903)

Markiana nigripinnis (Perugia, 1891)

Metynnis lippincottianus (Cope, 1870)

Metynnis maculatus (Kner, 1858)

Metynnis mola Eigenmann \& Kennedy, 1903

Moenkhausia bonita Benine et al., 2004

Moenkhausia cf. gracilima Eigenmann, 1908

Moenkhausia dichroura (Kner, 1858)

Moenkhausia forestii Benine et al., 2009

Moenkhausia intermedia Eigenmann, 1908

Moenkhausia lopesi Britski \& de Silimon, 2001

Moenkhausia oligolepis (Günther, 1864)

Moenkhausia sanctaefilomenae (Steindachner, 1907)

Myleus levis Eigenmann \& McAtee, 1907

Myleus tiete (Eigenmann \& Norris, 1900) *

Mylossoma duriventre (Cuvier, 1818)

Odontostilbe paraguayensis Eigenmann \& Kennedy, 1903

Odontostilbe pequira (Steindachner, 1882)

Oligosarcus paranensis Menezes \& Géry, 1983

Oligosarcus perdido Ribeiro et al., 2007

Oligosarcus pintoi Amaral Campos, 1945

Oligosarcus planaltinae Menezes \& Géry, 1983

Phenacogaster tegatus (Eigenmann, 1911)

Piabarchus analis (Eigenmann, 1914)

Piabarchus torrenticola Mahnert \& Géry, 1988

Piabina argentea Reinhardt, 1867

Piabucus melanostomus Holmberg, 1891

Piaractus mesopotamicus (Holmberg, 1887)

Poptella paraguayensis (Eigenmann, 1907)

Prionobrama paraguayensis (Eigenmann, 1914)

Psellogrammus kennedyi (Eigenmann, 1903)

Pygocentrus nattereri Kner, 1858

Roeboides affinis (Steindachner, 1879)

Roeboides descalvadensis Fowler, 1932

Roeboides microlepis (Reinhardt, 1851)

Salminus brasiliensis (Cuvier, 1816)

Salminus hilarii Valenciennes, 1850

Serrapinnus calliurus (Boulenger, 1900)

Serrapinnus heterodon (Eigenmann, 1915)

Serrapinnus kriegi (Schindler, 1937)

Serrapinnus microdon (Eigenmann, 1915)

Serrapinnus notomelas (Eigenmann, 1915)

Serrasalmus maculatus Kner, 1858

Serrasalmus marginatus Valenciennes, 1837

Alto Paraná

Paraguai

$+$

$+$

Tetragonopterus argenteus Cuvier, 1816

Triportheus nematurus (Kner, 1858)

Triportheus pantanensis Malabarba, 2004

Xenurobrycon macropus Myers \& Miranda Ribeiro, 1945

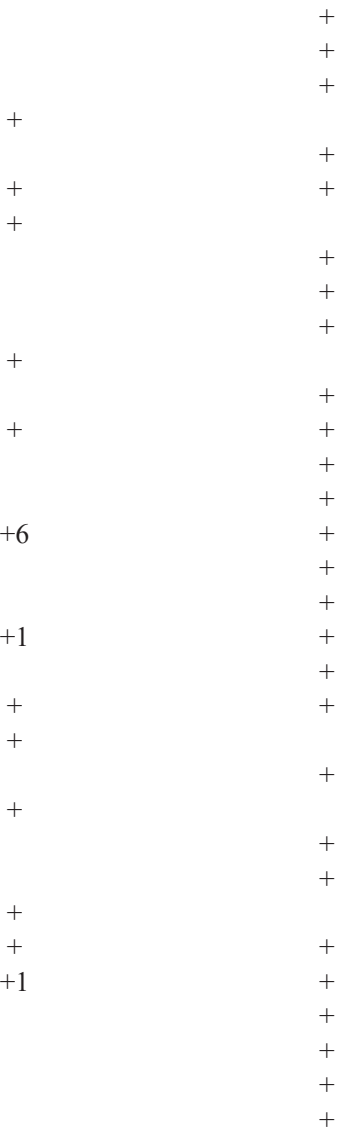


Tab. I. Cont.

Crenuchidae

Alto Paraná

Paraguai

Characidium gomesi Travassos, 1956

Characidium laterale (Boulenger, 1895)

Characidium cf. zebra Eigenmann, 1909

Curimatidae

Curimatella dorsalis (Eigenmann \& Eigenmann, 1889)

Curimatopsis myersi Vari, 1982

Cyphocharax gillii (Eigenmann \& Kennedy, 1903)

Cyphocharax modestus (Fernández-Yepez, 1948)

Cyphocharax nagelii (Steindachner, 1881)

Cyphocharax vanderi (Britski, 1980)

Potamorhina squamoralevis (Braga \& Azpelicueta, 1983)

Psectrogaster curviventris Eigenmann \& Kennedy, 1903

Steindachnerina brevipinna (Eigenmann \& Eigenmann, 1889)

Steindachnerina conspersa (Holmberg, 1891)

Steindachnerina insculpta (Fernández-Yepez, 1948)

Steindachnerina nigrotaenia (Boulenger, 1902)

Cynodontidae

Rhaphiodon vulpinus Spix \& Agassiz, 1829

Erythrinidae

Erythrinus erythrinus (Bloch \& Schneider, 1801)

Hoplerythrinus unitaeniatus (Spix \& Agassiz, 1829)

Hoplias intermedius (Günther, 1864)

Hoplias malabaricus (Bloch, 1794)

Gasteropelecidae

Gasteropelecus sternicla (Linnaeus, 1758)

Thoracocharax stellatus (Kner, 1858)

Hemiodontidae

Hemiodus orthonops Eigenmann \& Kennedy, 1903

Hemiodus semitaeniatus Kner, 1858

Lebiasinidae

Pyrrhulina australis Eigenmann \& Kennedy, 1903

Parodontidae

Apareiodon affinis (Steindachner, 1879)

Apareiodon ibitiensis Amaral Campos, 1944

Apareiodon piracicabae (Eigenmann, 1907)

Parodon nasus Kner, 1859

Prochilodontidae

Prochilodus lineatus (Valenciennes, 1837)

Gymnotiformes

Apteronotidae

Apteronotus acidops Triques, 2011

Apteronotus albifrons (Linnaeus, 1766)

Apteronotus bonapartii (Castelnau, 1855)

Apteronotus brasiliensis (Reinhardt, 1852)

Apteronotus caudimaculosus de Santana, 2003

Apteronotus ellisi (Alonso de Arámburu, 1957)

Sternarchorhynchus britskii Campos-da-Paz, 2000*

Tembeassu marauna Triques, 1988

Gymnotidae

Gymnotus inaequilabiatus (Valenciennes, 1839)

Gymnotus pantanal Fernandes et al., 2005

Gymnotus paraguensis Albert \& Crampton, 2003

Gymnotus sylvius Albert \& Fernandes-Matioli, 1999

$+$

$+$

$+$

$+1,3$

$+$

$+$

$+$

$+$

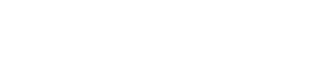

$+1$

$+$

$+$

$+1$

$+$

Rhamphichthys hahni (Meinken, 1937)

Sternopygidae

Eigenmannia trilineata López \& Castello, 1966

Eigenmannia virescens (Valenciennes, 1836)

Sternopygus macrurus (Bloch \& Schneider, 1801)

Siluriformes

Aspredinidae
$+$

$+$

$+$

(1)

(1)

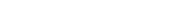

$+1$

$+$

$+$ 
Tab. I. Cont.

Amaralia hypsiura (Kner, 1855)

Bunocephalus doriae Boulenger, 1902

Bunocephalus larai Ihering, 1930

Pseudobunocephalus rugosus (Eigenmann \& Kennedy, 1903)

Auchenipteridae

Ageneiosus inermis (Linnaeus, 1766)

Ageneiosus militaris Valenciennes, 1835

Ageneiosus ucayalensis Castelnau, 1855

Auchenipterus nigripinnis (Boulenger, 1895)

Auchenipterus osteomystax (Miranda Ribeiro, 1918)

Entomocorus radiosus Reis \& Borges, 2006

Epapterus dispilurus Cope, 1878

Tatia neivai (Ihering, 1930)

Trachelyopterus coriaceus Valenciennes, 1840

Trachelyopterus galeatus (Linnaeus, 1766)

Trachelyopterus striatulus (Steindachner, 1877)

Alto Paraná

Paraguai

Clichthyidae

Aspidoras fuscoguttatus Nijssen \& Isbrücker, 1976

Brochis britskii Nijssen \& Isbrücker, 1983

Brochis splendens (Castelnau, 1855)

Callichthys callichthys (Linnaeus, 1758)

Corydoras aeneus (Gill, 1858)

Corydoras areio Knaack, 2000

Corydoras aurofrenatus Eigenmann \& Kennedy, 1903

Corydoras hastatus Eigenmann \& Eigenmann, 1888

Corydoras latus Pearson, 1924

Corydoras pantanalensis Knaack, 2001

Corydoras polystictus Regan, 1912

Hoplosternum littorale (Hancock, 1828)

Lepthoplosternum pectorale (Boulenger, 1895)

Megalechis thoracata (Valenciennes, 1840)

Cetopsidae

Cetopsis gobioides Kner, 1858

Clariidae

Clarias gariepinus (Burchell, 1822)

Doradidae

Anadoras weddellii (Castelnau, 1855)

Merodoras nheco Higuchi et al., 2007

Ossancora eigenmanni (Boulenger, 1895)

Ossancora punctata (Kner, 1853)

Oxydoras kneri Bleeker, 1862

Platydoras armatulus (Valenciennes, 1840)

Pterodoras granulosus (Valenciennes, 1821)

Rhinodoras dorbignyi (Kner, 1855)

Trachydoras paraguayensis (Eigenmann \& Ward, 1907)

Cetopsorhamdia iheringi Schubart \& Gomes, 1959

Imparfinis borodini Mees \& Cala, 1989

Imparfinis mirini Haseman, 1911

Imparfinis schubarti (Gomes, 1956)

Imparfinis stictonotus (Fowler, 1940)

Phenacorhamdia hoehnei (Miranda Ribeiro, 1914)

Phenacorhamdia tenebrosa (Schubart, 1964)

Pimelodella avanhandavae Eigenmann, 1917

Pimelodella gracilis (Valenciennes, 1835)

Pimelodella mucosa Eigenmann \& Ward, 1907

Pimelodella taenioptera Miranda Ribeiro, 1914

Rhamdia quelen (Quoy \& Gaimard, 1824)

Ictaluridae

Ictalurus punctatus (Rafinesque, 1818)

Loricariidae

Ancistrus cuiabae Knaack, 1999

Corumbataia britskii Ferreira \& Ribeiro, 2007

Farlowella amazona (Günther, 1864)

Farlowella hahni Meinken, 1937

Farlowella isbrueckeri Retzer \& Page, 1997
$+1$

Paraná Parag

$+1$

+
+1

$+1$

$+1$

$+$

(n)

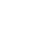


Tab. I. Cont.

Farlowella jauruensis Eigenmann \& Vance, 1917

Farlowella paraguayensis Retzer \& Page, 1997

Hemiodontichthys acipenserinus (Kner, 1853)

Pimelodidae

Alto Paraná

Paraguai

Hisonotus francirochai (Ihering, 1928)

Hisonotus insperatus Britski \& Garavello, 2003

Hypoptopoma inexspectatum (Holmberg, 1893)

Hypostomus albopunctatus (Regan, 1908)

Hypostomus ancistroides (Ihering, 1911)

Hypostomus boulengeri (Eigenmann \& Kennedy, 1903)

Hypostomus cochliodon Kner, 1854

Hypostomus denticulatus Zawadzki et al., 2008

Hypostomus hermanni (Ihering, 1905)

Hypostomus iheringii (Regan, 1908)

Hypostomus latifrons Weber, 1986

Hypostomus latirostris (Regan, 1904)

Hypostomus margaritifer (Regan, 1908)

Hypostomus nigromaculatus (Schubart, 1964)

Hypostomus piratatu Weber, 1986

Hypostomus regani (Ihering, 1905)

Hypostomus strigaticeps (Regan, 1908)

Hypostomus topavae (Godoy, 1969)

Loricaria coximensis Rodriguez et al., 2012

Loricaria lentiginosa Isbrücker, 1979

Loricaria simillima Regan, 1904

Loricariichthys labialis (Boulenger, 1895)

Loricariichthys platymetopon Isbrücker \& Nijssen, 1979

Loricariichthys rostratus Reis \& Pereira, 2000

Megalancistrus parananus (Peters, 1881)

Neoplecostomus paranensis Langeani, 1990

Otocinclus bororo Schaefer, 1997

Otocinclus mariae Fowler, 1940

Otocinclus vittatus Regan, 1904

Otothyropsis polyodon Calegari et al., 2013

Proloricaria prolixa (Isbrücker \& Nijssen, 1978)

Pseudohemiodon platycephalus (Kner, 1853)

Pterygoplichthys ambrosettii (Holmberg, 1893)

Pyxiloricaria menezesi Isbrücker \& Nijssen, 1984

Rhinelepis aspera Spix \& Agassiz, 1829

Rineloricaria aurata (Knaack, 2003)

Rineloricaria cacerensis (Miranda Ribeiro, 1912)

Rineloricaria lanceolata (Günther, 1868)

Rineloricaria latirostris (Boulenger, 1900)

Rineloricaria parva (Boulenger, 1895)

Spatuloricaria evansii (Boulenger, 1892)

Sturisoma barbatum (Kner, 1853)

Sturisoma robustum (Regan, 1904)

Hemisorubim platyrhynchos (Valenciennes, 1840)

Hypophthalmus edentatus Spix \& Agassiz, 1829

Iheringichthys labrosus (Lütken, 1874)

Iheringichthys syi Azpelicueta \& Britski 2012

Leiarius pictus (Müller \& Troschel, 1849)

Luciopimelodus pati (Valenciennes, 1835)

Megalonema platanum (Günther, 1880)

Pimelodus absconditus Azpelicueta, 1995

Pimelodus argenteus Perugia, 1891

Pimelodus maculatus La Cepède, 1803

Pimelodus microstoma Steindachner, 1877

Pimelodus mysteriosus Azpelicueta, 1998

Pimelodus ornatus Kner, 1858

Pimelodus pantaneiro Souza-Filho \& Shibatta, 2007

Pimelodus paranensis Britski \& Langeani, 1988

Pinirampus pirinampu (Spix \& Agassiz, 1829)

Pseudoplatystoma corruscans (Spix \& Agassiz, 1829)

Pseudoplatystoma reticulatum Eigenmann \& Eigenmann, 1889

Sorubim lima (Bloch \& Schneider, 1801)
$+$

$+$

$+$

$+1$

$+$

$+$

$+$

$+$

$+$

$+$

$+$

$+$

$+$

+
+
+


Tab. I. Cont.

\begin{tabular}{|c|c|c|}
\hline & Alto Paraná & Paraguai \\
\hline Zungaro jahu (Ihering, 1898) & + & + \\
\hline \multicolumn{3}{|l|}{ Pseudopimelodidae } \\
\hline Pseudopimelodus mangurus (Valenciennes, 1835) & + & + \\
\hline Pseudopimelodus pulcher (Boulenger, 1887) & + & + \\
\hline \multicolumn{3}{|l|}{ Scoloplacidae } \\
\hline Scoloplax dicra Bailey \& Baskin, 1976 & & + \\
\hline Scoloplax empousa Schaefer et al., 1989 & + & + \\
\hline \multicolumn{3}{|l|}{ Trichomycteridae } \\
\hline Ituglanis eichorniarum (Miranda Ribeiro, 1912) & & + \\
\hline Ituglanis herberti (Miranda Ribeiro, 1940) & & + \\
\hline Ochmacanthus batrachostoma (Miranda Ribeiro, 1912) & & + \\
\hline Parastegophilus maculatus (Steindachner, 1879) & & + \\
\hline Paravandellia oxyptera Miranda Ribeiro, 1912 & + & + \\
\hline Trichomycterus cf. diabolus Bockmann et al., 2004 & + & \\
\hline \multicolumn{3}{|l|}{ Cyprinodontiformes } \\
\hline \multicolumn{3}{|l|}{ Poeciliidae } \\
\hline Pamphorichthys hasemani (Henn, 1916) & & + \\
\hline Pamphorichthys hollandi (Henn, 1916) & + & \\
\hline Phalloceros harpagos Lucinda, 2008 & + & \\
\hline Phallotorynus pankalos Lucinda et al., 2005 & + & \\
\hline Phallotorynus victoriae Oliveros, 1983 & + & \\
\hline Poecilia reticulata Peters, 1859 & +4 & +4 \\
\hline \multicolumn{3}{|l|}{ Rivulidae } \\
\hline Melanorivulus apiamici (Costa, 1989) & + & \\
\hline Melanorivulus dapazi (Costa, 2005) & & + \\
\hline Melanorivulus egens (Costa, 2005) & + & \\
\hline Melanorivulus pictus (Costa, 1989) & + & \\
\hline Melanorivulus punctatus (Boulenger, 1895) & & + \\
\hline Melanorivulus rossoi (Costa, 2005) & + & \\
\hline Melanorivulus scalaris (Costa, 2005) & + & \\
\hline Melanorivulus vittatus (Costa, 1989) & + & \\
\hline Neofundulus paraguayensis (Eigenmann \& Kennedy, 1903) & & + \\
\hline Pterolebias longipinnis Garman, 1895 & & + \\
\hline Pterolebias phasianus Costa, 1988 & & + \\
\hline Stenolebias bellus Costa, 1995* & & + \\
\hline Trigonectes balzanii (Perugia, 1891) & & + \\
\hline \multicolumn{3}{|l|}{ Beloniformes } \\
\hline \multicolumn{3}{|l|}{ Belonidae } \\
\hline Potamorrhaphis eigenmanni Miranda Ribeiro, 1915 & & + \\
\hline \multicolumn{3}{|l|}{ Synbranchiformes } \\
\hline \multicolumn{3}{|l|}{ Synbranchidae } \\
\hline Synbranchus marmoratus Bloch, 1795 & + & + \\
\hline \multicolumn{3}{|l|}{ Perciformes } \\
\hline \multicolumn{3}{|l|}{ Cichlidae } \\
\hline Aequidens plagiozonatus Kullander, 1984 & & + \\
\hline Apistogramma borellii (Regan, 1906) & & + \\
\hline Apistogramma commbrae (Regan, 1906) & +6 & + \\
\hline Apistogramma inconspicua Kullander, 1983 & & + \\
\hline Apistogramma trifasciata (Eigenmann \& Kennedy, 1903) & & + \\
\hline Astronotus crassipinnis (Heckel, 1840) & +5 & + \\
\hline Bujurquina vittata (Heckel, 1840) & & + \\
\hline Chaetobranchopsis australis Eigenmann \& Ward, 1907 & & + \\
\hline Cichla kelberi Kullander \& Ferreira, 2006 & +2 & \\
\hline Cichla piquiti Kullander \& Ferreira, 2006 & +2 & +2 \\
\hline Cichlasoma dimerus (Heckel, 1840) & & + \\
\hline Cichlasoma paranaense Kullander, 1983 & + & \\
\hline Crenicichla britskii Kullander, 1982 & + & \\
\hline Crenicichla haroldoi Luengo \& Britski, 1974 & + & \\
\hline Crenicichla jaguarensis Haseman, 1911 & + & \\
\hline Crenicichla lepidota Heckel, 1840 & & + \\
\hline Crenicichla semifasciata (Heckel, 1840) & & + \\
\hline Crenicichla vittata Heckel, 1840 & & + \\
\hline Geophagus cf. proximus (Castelnau, 1855) & +5 & \\
\hline Gymnogeophagus balzanii (Perugia, 1891) & & + \\
\hline Gymnogeophagus setequedas Reis et al., 1992* & + & \\
\hline Laetacara dorsigera (Heckel, 1840) & & + \\
\hline
\end{tabular}


Tab. I. Cont.

\begin{tabular}{|c|c|c|}
\hline & Alto Paraná & Paraguai \\
\hline Mesonauta festivus (Heckel, 1840) & & + \\
\hline Oreochromis niloticus (Linnaeus, 1758) & +2 & \\
\hline Satanoperca pappaterra $($ Heckel, 1840$)$ & $+1,2$ & + \\
\hline Tilapia rendalli (Boulenger, 1897) & +2 & +2 \\
\hline \multicolumn{3}{|l|}{ Sciaenidae } \\
\hline Pachyurus bonariensis Steindachner, 1879 & & + \\
\hline Plagioscion squamosissimus (Heckel, 1840) & +2 & \\
\hline Plagioscion ternetzi Boulenger, 1895 & & + \\
\hline \multicolumn{3}{|l|}{ Pleuronectiformes } \\
\hline \multicolumn{3}{|l|}{ Achiridae } \\
\hline Catathyridium jenynsii (Günther, 1862) & +1 & + \\
\hline \multicolumn{3}{|l|}{ Lepidosireniformes } \\
\hline \multicolumn{3}{|l|}{ Lepidosirenidae } \\
\hline Lepidosiren paradoxa Fitzinger, 1837 & & + \\
\hline${ }^{2}$ & 201 & 255 \\
\hline
\end{tabular}

esquerda do rio Paraguai, até próximo à cidade de Corumbá. Uma espécie de corvina (Plagioscion squamosissimus) foi introduzida no estado de São Paulo em meados da década de 1960 (HAHN et al., 1997) e posteriormente em outros locais (e.g. BENNEMANN et al., 2006), visando a pesca comercial. Dois caracídeos amazônicos foram registrados na vertente do Alto Paraná em Mato Grosso do Sul: Metynnis lippincottianus e Hyphessobrycon moniliger. Para a primeira espécie existem lotes (NUP) do sistema do rio Ivinhema, ao sul, ao reservatório de Jupiá, no norte do estado; sua presença na bacia permanece sem explicação (GRAÇA \& PAVANELLI, 2007; JúLIO JR. et al., 2009). A segunda foi registrada em dois córregos, afluentes da margem direita do reservatório de Ilha Solteira (DZSJRP008858 e DZSJRP009000); os dois lotes contem várias dezenas de indivíduos, sugerindo populações instaladas localmente. A origem poderia ser por uso como ornamental, mas uma busca em sites de aquariofilia não mostra que a espécie esteja sendo explorada, mesmo que seu uso seja permitido (IN MMA n ${ }^{13}$ ). LANGEANI et al. (2007) registraram Farlowella hahni, um loricariídeo originário do sistema do médio rio Paraná (Retzer \& PAGe, 1997), com base em um indivíduo coletado no rio Paraná, no lado de Mato Grosso do Sul (DZSJRP004409) e relacionaram sua presença à barragem de Itaipu.

As duas drenagens de Mato Grosso do Sul compartilham 100 espécies, $28 \%$ do total. Cerca de metade dessas espécies compartilhadas (49) ocorrem naturalmente nas duas bacias. Das outras espécies compartilhadas, três foram introduzidas nas duas vertentes (ver discussão no parágrafo anterior), as únicas introduzidas listadas para a drenagem do rio Paraguai no estado. Outras 45 espécies compartilhadas foram introduzidas na vertente do rio Paraná, a maioria (38) após a instalação da barragem da hidrelétrica de Itaipu, em 1982, e do canal da piracema, vinte anos depois (GRAÇA \& PAVANELli, 2007; LANGEANi et al., 2007 e JúLio JR. et al., 2009). Quase um terço (64) das 201 espécies registradas para a bacia do Alto Paraná no Mato Grosso do Sul são espécies introduzidas. LANGEANI et al. (2007) registraram percentual de $22,9 \%$ de espécies introduzidas para toda a bacia do Alto Paraná. Com a melhora do conhecimento ainda imperfeito da ictiofauna da região que virá com o projeto BIOTA-MS, deve diminuir a proporção de espécies introduzidas em Mato Grosso do Sul, mesmo que seu número absoluto aumente, uma expectativa razoável.

É necessário tecer alguns comentários sobre alguns dos nomes que aparecem, ou não, na Tabela I. Apenas uma espécie de Ancistrus foi incluída: A. cuiabae. Ancistrus formoso, espécie troglóbia, ocorre na drenagem subterrânea do Planalto da Bodoquena (CORDEIRo et al., 2014). Indivíduos do gênero são bastante comuns, podendo ser coletados em riachos das cabeceiras das duas bacias hidrográficas. Os lotes depositados em algumas coleções (ZUFMS-PIS, NUP e LIRP, quase 200 lotes) são sempre provenientes de riachos que apresentam ao menos alguns trechos ainda não assoreados, com substrato rochoso. Determinar as espécies a que esse material pertence é tarefa difícil, já que as descrições mais antigas são no geral vagas e nossos espécimes não se conformam às espécies descritas mais recentemente (exceto a espécie citada acima, que parece habitar as planícies de inundação em terras mais baixas, não os riachos do planalto - ver localidades em KNAACK (1999) e MARIOTTO et al. (2009). O gênero tem mais de 60 espécies descritas, mas uma revisão completa faz-se necessária (BIFI et al., 2009) antes que possamos identificar as espécies presentes em Mato Grosso do Sul com algum grau de acurácia.

Outro grupo de Loricariidae que é muito diversificado e apresenta problemas sistemáticos é o gênero Hypostomus. Segundo Weber (2003), trata-se um dos mais complexos e diversificados grupos de Siluriformes neotropicais, devido a descrições incompletas, falta de informações sobre padrões de distribuição e à grande variabilidade intraespecífica. Os Hypostomus da bacia Paraná-Paraguai vêm á sendo estudados há algum tempo (e.g. ZAWADZKY et al., 1999, 2002, 2008, 2010, 2014; JerEP et al., 2007; MARTIns et al., 2012), de modo que foi possível listar várias espécies para o estado. Estudos em andamento indicaram a existência de seis formas novas para a ciência na bacia do Alto Paraguai em MS, bacia esta que contém uma diversidade ainda não descrita para o gênero (ZAWADZKY et al., 2010); duas descrições já foram submetidas para publicação.

Alguns nomes incluídos na lista representam formas 
que pertencem a grupos de espécies ainda não resolvidos. Esses nomes eram tradicionalmente usados para as formas em questão. Optamos por incluir essas formas, com os epítetos antigos, na listagem da Tabela I porque são espécies ubíquas no estado. Dessa maneira, o leitor tem mais precisão a respeito de qual(is) táxon(s) foram registrados, ao invés de numerá-los com números arábicos (e.g. Astyanax sp. 1). Enquadram-se nesta categoria Astyanax fasciatus e A. paranae. Diversos autores, baseados em dados morfológicos e cromossômicos, afirmam que Astyanax fasciatus é, na verdade, um complexo de espécies. Melo (2005) restringiu a distribuição desta espécie a Bacia do Rio São Francisco e afirmou que os espécimes similares a $A$. fasciatus oriundos da Bacia do Rio Paraná são, de fato, um grupo de espécies. MaIstro et al. (1998) detectaram diferenças cromossômicas entre as populações de Astyanax paranae, uma espécie de regiões de cabeceiras que pode representar outro complexo de espécies.

Outro reconhecido complexo de espécies é representado pelo que denominamos aqui de Hoplias malabaricus. GRAÇA \& PAVANELLI (2007) reconhecem três formas para as quais esse nome era aplicado, uma delas proveniente da bacia do Médio rio Paraná. Na bacia do Paraguai, os lotes depositados na ZUFMS-PIS permitem reconhecer duas formas, uma compatível com aquela introduzida na vertente do Alto Paraná.

Por se tratar de uma espécie amazônica, Moenkhausia gracilima, foi reportada aqui como $M$. cf. gracilima, seguindo GraÇA \& PAVANELli (2007).

A seguir algumas formas novas que estão em estudo: Astyanax sp. 1: Ricardo M.C. Castro (USP, Ribeirão Preto), Marcel R. Cavallaro (UNEB, Paulo Afonso), Otávio Froehlich (UFMS, Campo Grande), José Sabino (Anhanguera/UNIDERP, Campo Grande); Astyanax sp. 2: Marcel R. Cavallaro (UNEB, Paulo Afonso), Otávio Froehlich (UFMS, Campo Grande); Hypostomus sp. 1, 2, 3, 4: Cláudio H. Zawadzky (UEM), Luiz F.C. Tencatt (UEM), Otávio Froehlich (UFMS, Campo Grande); Ancistrus sp. 1, 2, 3: Cláudio H. Zawadzky (UEM), Luiz F.C. Tencatt (UEM), Otávio Froehlich (UFMS, Campo Grande); Loricaria sp.: Mónica Rodriguez (UFU), Marcel R. Cavallaro (UNEB, Paulo Afonso), Matthew R. Thomas (Kentucky Department of Fish and Wildlife Resources), Otávio Froehlich (UFMS, Campo Grande).

Ameaças e conservação da ictiofauna do Mato Grosso do Sul. De modo geral, a manutenção do regime de escoamento de águas e a ausência de contaminantes são cruciais para a integridade dos ecossistemas aquáticos. De maneira específica, distúrbios causados pela atividade do homem podem impactar negativamente os componentes vivos dos ambientes aquáticos, tanto na bacia do Paraná como na Bacia do Alto Paraguai - BAP.

Características físicas, tais como geomorfologia do alto rio Paraguai, com planaltos circundantes onde as nascentes dos rios estão localizadas, regulam e moldam a inundação sazonal da planície pantaneira. A regularidade do pulso de inundação dos hábitats do Pantanal favorece a manutenção da diversidade e da abundância dos organismos, bem como de seus papeis ecológicos no bioma (ALHO, 2005; HARris et al., 2005).

No cenário atual, há evidências de crescentes pressões que, em futuro próximo, poderão afetar negativamente a biodiversidade aquática (HARRIS et al., 2005; CALHEIROS et al., 2009; AlHo \& SABINO, 2011). Alguns desses distúrbios são resultados da ação direta sobre o fluxo do rio, decorrentes da instalação de pequenas centrais hidrelétricas, outros são influências indiretas associadas às mudanças de uso da terra e do potencial de impacto derivado de mudanças na legislação ambiental, que poderá promover desmatamentos no planalto, onde nascem os rios que alimentam o Pantanal e porções da bacia do Paraná dentro do Mato Grosso do Sul.

A conversão de vegetação natural em pastagens e culturas agrícolas tem sido drástica nas últimas décadas, nos planaltos do Brasil Central. Monitoramento da vegetação e uso da terra na parte brasileira da BAP, considerando o período de 2002 a 2008, mostrou que a região sofreu consequências da expansão da pecuária e atividade agrícola, especialmente na região do planalto $(\mathrm{CI}+\mathrm{ECOA}+\mathrm{AVINA}$ + SOS Pantanal + WWF-Brasil, 2009). Esse estudo aponta que, embora o Pantanal mantenha perto de $85 \%$ de sua vegetação natural, os planaltos circundantes registraram a presença de $41,8 \%$ de sua cobertura vegetal original. Durante o curto período de 2002 a 2008, a BAP perdeu 4\% de sua vegetação nos planaltos e $2,4 \%$ em área de várzea. Os dados para 2008 mostraram, ainda, que a pecuária é responsável por $11,1 \%$ de conversão de vegetação pantaneira e 43,5\% de alteração nos planaltos do entorno.

O potencial de alteração do fluxo dos rios por usinas hidrelétricas é digno de nota para todo o Mato Grosso do Sul. Mesmo que a maioria das usinas a serem instaladas ou com potencial de instalação - na região seja de pequeno porte (Pequenas Centrais Hidrelétricas, PCHs), o efeito de deletério, em conjunto, poderá resultar em um impacto importante. Em geral, as pequenas $\mathrm{PCHs}$, que não formam propriamente um reservatório, podem alterar a descarga de nutrientes e a matéria em suspensão, o que afeta a ciclagem de nutrientes em corpos d'água afetados. Além disso, a presença da barreira física formada pela barragem é um conhecido obstáculo para o deslocamento de peixes migradores durante a época da desova, que afeta a reprodução das espécies no médio e longo prazos (CALHEIRos et al., 2009; FERnANDES et al., 2010).

Cerca de $70 \%$ da água do Pantanal têm origem na parte norte da bacia, em especial da sub-bacia do Rio Cuiabá, o principal afluente do Pantanal que contribui com $40 \%$ para o sistema (MMA, 1997). Embora exista a informação de que $75 \%$ de todos os 115 projetos de barragens planejadas para a bacia do Alto Paraguai (BAP) estejam na Região Norte, em Mato Grosso (ANEEL, 2010), é possível vislumbrar um cenário preocupante, uma vez que todos esses acontecimentos podem mudar a hidrodinâmica e inundações na planície pantaneira como um todo (GIRARD, 2002).

Com as barragens instaladas, para que haja a conservação de parcela da fauna de peixes, particularmente das espécies migratórias, é mandatório que exista a 
conectividade entre as partes a montante e a jusante das usinas, mantidas também as conexões entre o canal do rio e suas áreas de inundação marginais (CALHeIros et al., 2009). Todas essas mudanças e impactos negativos sobre a ecologia dos ecossistemas de cada sub-bacia formadora do Pantanal devem ser avaliados simultaneamente, antes da instalação desses projetos na BAP (CALHEIRos et al., 2009). Tais preocupações devem ser ampliadas ao sistema do rio Paraná.

Na história recente, o uso e ocupação humana nos hábitats do Mato Grosso do Sul têm facilitado a introdução de espécies invasoras de plantas e animais aquáticos, incluindo espécies domésticas. Espécies exóticas ameaçam a biodiversidade regional, uma vez que modificam a estrutura da comunidade, alteram hábitats naturais e afetam a biodiversidade local. A União Internacional para a Conservação da Natureza (IUCN) e o governo brasileiro identificam as espécies invasoras como a terceira ameaça mais importante para a biodiversidade, seguindo perda de hábitat e efeito direto sobre a espécie. Além disso, espécies exóticas podem introduzir patógenos ou funcionar como vetores ou reservatórios de doenças que afetam a biota aquática (ALHO, 2005; Alho \& Sabino, 2011).

Além de alterações ambientais e distúrbios de hábitats, pressões de pesca sobre espécies de peixes de maior porte, com interesse econômico e esportivo, podem afetar negativamente tais populações. Dourado (Salminus brasiliensis), pintando (Pseudoplatystoma corruscans), cachara (Pseudoplatystoma reticulatum), curimbatá (Prochilodus lineatus), pacu (Piaractus mesopotamicus), piraputanga (Brycon hilarii) e piavuçu (Leporinus friderici) são algumas das espécies mais notáveis da ictiofauna do Pantanal. Isso é devido, em parte, ao tamanho dessas espécies de peixes grandes, valiosas para a pesca amadora e profissional (CATELlA, 2004). Menos evidente e menos conhecidos, mas não menos importante, são as espécies de peixes de pequeno porte, de até $15 \mathrm{~cm}$ de comprimento. Sem os pequenos caracídeos, bagres pequenos e cascudinhos, muitas espécies de grande porte não poderiam existir: pequenas espécies aquáticas compõem diretamente ou são elos das cadeias alimentares das espécies maiores. Essencialmente, os peixes de pequeno porte são organismos cuja riqueza biológica ainda está para ser devidamente avaliada, especialmente nas cabeceiras do Pantanal (SABINo \& PRADO, 2006).

Outra ameaça à ictiofauna é a poluição das águas. A introdução de toxinas e outros contaminantes nos sistemas aquáticos do Mato Grosso do Sul é uma tendência indesejável para manutenção da qualidade dos hábitats, uma vez que afeta parcelas mais sensíveis da ictiofauna, bem como a qualidade dos recursos naturais, incluindo águas superficiais e subterrâneas (ALHo \& VIEIRA, 1997).

O turismo - se bem planejado e regulado - oferece alternativas econômicas e potencial de geração de renda e emprego para a região. Há um trade turístico em franca expansão no Pantanal e em áreas do rio Paraná. Infelizmente, a maior parte desse turismo é predatória. Grupos turísticos não controlados podem invadir áreas pristinas que deveriam ser preservadas (por exemplo, ninhais e áreas de reprodução de peixes), pescar mais peixe que o permitido, ou ainda espalhar lixo. Numerosos barcos de turismo atuam como hotéis flutuantes em todo o Pantanal e, em geral, não tratam os resíduos de forma adequada, permitindo que se espalhem resíduos em seu caminho (AlHo, 2005).

De outro lado, o turismo de observação da vida selvagem nos ambientes naturais do Mato Grosso do Sul pode atender a demandas sociais e econômicas de diferentes regiões, mas não poderá suprimir os cuidados com a operação de mínimo impacto. O turismo de mínimo impacto como o praticado em Bonito pode oferecer lições de convívio harmônico associado à sustentabilidade econômica e ambiental genuínas (SABINo et al., 2012). A atividade pode inspirar adaptações a diferentes realidades do estado, incluir a participação das comunidades locais, promover articulação de gestores de área protegidas e fomentar a coesão entre os setores público e privado, com importante papel na popularização da ciência e na sensibilização da sociedade para a e conservação da vida selvagem. Combinado com esforços para a educação ambiental, incluindo a construção de instalações para visitantes, como um aquário público, a atividade turística pode melhorar a estrutura e promover a interpretação ambiental (SABINO et al., 2012).

Finalmente, para minimizar toda sorte de pressões à ictiofauna, é necessário fortalecer institucionalmente as diversas instâncias de fiscalização e aplicação da lei no Mato Grosso do Sul. A fragilidade de políticas de gestão e falta de pessoal qualificado são identificadas como elementos importantes que contribuem para a degradação ambiental. Assim, três elementos precisam ser reforçados para melhorar a gestão participativa dos sistemas aquáticos na região: (1) fortalecer o conhecimento dos tomadores de decisão sobre os recursos hídricos do Mato Grosso do Sul, em especial do Pantanal, (2) ampliar as competências dos interessados em usar os recursos de maneira sustentável, e (3) a motivar os moradores para participar de decisões que afetam diretamente os ecossistemas aquáticos (modificado de AlHo \& SABINO, 2011).

Agradecimentos. A Fundação de Apoio ao Desenvolvimento do Ensino, Ciências e Tecnologia do Estado de Mato Grosso do Sul (Fundect) e a Superintendência de Ciências e Tecnologia do Estado de Mato Grosso do Sul (Sucitec/MS) pelo convite de participação neste fascículo especial da Iheringia, Série Zoologia e o suporte financeiro para sua publicação. Vários colegas deram contribuições fundamentais para a elaboração da lista de espécies: Carla Simone Pavanelli, Flávio Bockmann, Francisco Langeani, João Paulo Capretz, Katiane Ferreira, Lilian Casatti, Luiz Fernando Caserta Tencatt, Murilo Carvalho, Verônica Slobodian e Weferson Júnio da Graça. Eles participaram seja com listagens de espécies dos grupos que estudam, tirando dúvidas quanto à identificação de lotes depositados em coleções e/ou na determinação da propriedade do uso de epítetos específicos para a região. Na ZUFMS-PIS o apoio de Francisco de Paula Severo da Costa Neto foi inestimável, na checagem de lotes e na triagem e identificação de material ainda não tombado. 


\section{REFERÊNCIAS BIBLIOGRÁFICAS}

Alho, C. J. R. 2005. The Pantanal. In: Fraser, L. H. \& Keddy, P. A. org. The world's largest wetlands - Ecology and Conservation. New York, Cambridge University Press, p.203-271.

Alho, C. J. R. \& Sabino, J. 2011. A conservation agenda for the Pantanal's biodiversity. Brazilian Journal of Biology 71(1 suppl.):327-335.

Alho, C. J. R. \& Vieira, L. M. 1997. Fish and wildlife resources in the Pantanal wetlands of Brazil and potential disturbances from the release of environmental contaminants. Environmental Toxicology and Chemistry 16(1):71-74.

ANEEL - Agência Nacional de Energia Elétrica. 2010. Quadro de acompanhamento de autorizações das PCH's. Ministério de Minas e Energia. Disponível em: <http://www.aneel.gov.br/ aplicacoes/autorizacoes/default_aplicacao_acompanhamento. cfm?idacompanhamentotipo $=4>$. Acessado em: 15.11.2012.

Bennemann, S. T.; Capra, L. G.; Galves, W. \& Shibatta, O. A. 2006. Dinâmica trófica de Plagioscion squamosissimus (Perciformes, Sciaenidae) em trechos de influência da represa Capivara (rios Paranapanema e Tibagi). Iheringia, Série Zoologia 96(1):115-119.

Bifi, A. G.; Pavanelli, C. S. \& ZaWAdSKy, C. H. 2009. Three new species of Ancistrus Kner, 1854 (Siluriformes: Loricariidae) from the Rio Iguaçu basin, Paraná State, Brazil. Zootaxa 2275:41-59.

Britski, H. A.; Silimon, K. Z. S. \& Lopes, B. S. 2007. Peixes do Pantanal. Manual de identificação. 2ed. Brasília, Embrapa. 227p.

Buckup, P. A.; Menezes, N. A. \& Ghazzi, M. S. eds. 2007. Catálogo das espécies de peixes de água doce do Brasil. Rio de Janeiro, Museu Nacional.

Calheiros, D.; Arndt, E.; Rodriguez, E. A. \& Silva, M. C. A. 2009. Influências de usinas hidrelétricas no funcionamento hidro-ecológico do Pantanal Mato-Grossense - Recomendações. EMBRAPA. Documentos 102:1-21

Castagnoli, N. 1992. Piscicultura de água doce. Jaboticabal, FUNEP. 189 p.

Castro, R. M. C. \& Menezes, N. A. 1998. Estudo diagnóstico da diversidade de peixes do Estado de São Paulo. In: Joly, C. A. \& Bicudo, C. E. M. org. Biodiversidade do Estado de São Paulo, Brasil: síntese do conhecimento ao final do século XX. Vertebrados. vol.6. São Paulo, Programa BIOTA/FAPESP, p. 3-13.

Catella, A. C. 2004. A pesca no Pantanal Sul: situação atual e perspectivas. EMBRAPA Pantanal. Documentos 48:1-83.

Chandra, G.; Bhattacharjee, I.; Chatterjee, S. N. \& Ghosh, A. 2008 Mosquito control by larvivorous fish. Indian Journal of Medical Research 127:13-27.

CONSERVAÇÃO INTERNACIONAL - CI. 2009. Monitoramento das alterações da cobertura vegetal e uso do solo na Bacia do Alto Paraguai Porção Brasileira. Disponível em: $<$ http://assets.wwfbr.panda.org/ downloads/mapacoberturabaciaaltoparaguai_estudocompleto.pdf $>$. Acessado em 15.11.2012.

Cordeiro, L. M.; Borghezan, R. \& Trajano, E. 2014. Subterranean biodiversity in the Serra da Bodoquena karst area, Paraguay River basin, Mato Grosso do Sul, Southwestern Brazil. Biota Neotropica 14(3). http://dx.doi.org/10.1590/1676-06032014011414

DeAcon, A. E. 2010. The behavioural ecology of the Trinidadian guppy, Poecilia reticulata, as an invasive species. $\mathrm{PhD}$ Thesis. St. Andrews, University of St Andrews. Disponível em: $<$ http://research-repository. standrews.ac.uk/bitstream/10023/1689/6/AmyDeaconPhDThesis.PDF $>$. Acessado em 16.10.2012.

Espinola, L. A. 2009. Cichla kelberi, Kullander e Ferreira, 2006; um piscívoro introduzido na Planície de inundação do alto rio Paraná: aplicação do modelo INVASS. Tese de doutorado. Maringá, Universidade Estadual de Maringá.

Fernandes, I. M.; Machado, F. A. \& Penha, J. 2010. Spatial pattern of a fish assemblage in a seasonal tropical wetland: effects of habitat, herbaceous plant biomass, water depth, and distance from species sources. Neotropical Ichthyology 8(2):289-298.

FERRARIS JR., C. J. 2007. Checklist of catfishes, recent and fossil (Osteichthyes: Siluriformes), and catalogue of siluriform primary types. Zootaxa 1418:1-628.

Froehlich, O.; Vilela, M. J. A.; Cavallaro, M. R. \& Cordeiro, L. M. 2006. Inventário da Ictiofauna do Complexo Aporé-Sucuriú. In:
Pagotto, T. C. S. \& Souza, P. R. org. Biodiversidade do Complexo Aporé-Sucuriú: Subsídios à Conservação e Manejo do Bioma Cerrado. Campo Grande, EDUFMS, p.89-102.

Girard, P. 2002. Efeito cumulativo das barragens no pantanal. Mobilização para conservação das áreas úmidas do Pantanal e Bacia do Araguaia. Campo Grande, MS: Instituto Centro Vida. Disponível em: <http://www.riosvivos.org.br/downloads/Efeito_cumulativo_ barragens Pantanal.pdf $>$. Acessado em: 15.11. 2012.

GraçA, W. J. \& Pavanelli, C. S. 2007. Peixes da planície de inundação do Alto rio Paraná e áreas adjacentes. Maringá, EDUEM. 241p.

Hahn, N. S.; Agostinho, A. A. \& Goitein, R. 1997. Feeding ecology of curvina Plagioscion squamosissimus (Hechel, 1840) (Osteichthyes, Perciformes) in the Itaipu Reservoir and Porto Rico floodplain (PR, Brazil). Acta Limnologica Brasiliensia 9:11-22.

Harris, M. B.; Tomas, W. M.; Mourão, G. M.; Da Silva, C. J.; Guimarães, E.; SonOdA, F. \& FACHIM, E. 2005. Desafios para proteger o Pantanal brasileiro: ameaças e iniciativas em conservação. Megadiversidade 1(1): 156-164.

Jerep, F. C. \& Shibatta, O. A. \& Zawadzky, C. H. 2007. A new species of Hypostomus Lacépède, 1803 (Siluriformes: Loricariidae) from the upper rio Paraná basin, Southern Brazil. Neotropical Ichthyology 5(4):435-442

Júlio JR., H. F.; Tós, C. D.; Agostinho, A. A. \& Pavanelli, C. S. 2009. A massive invasion of fish species after eliminating a natural barrier in the upper rio Paraná basin. Neotropical Ichthyology 7(4):709-718.

KnAACK, J. 1999. New Ancistrus species from the Rio Cuiaba System, Brazil (Pisces, Siluriformes, Loricariidae). Tropical Fish Hobbyist 47(12):150-155.

Langeani, F.; Castro, R. M. C.; Oyakawa, O. T.; Shibatta, O. A.; Pavanelli, C. S. \& Casatti, L. 2007. Diversidade da ictiofauna do Alto Rio Paraná: composição atual e perspectivas futuras. Biota Neotropica 7(3):181-197. Disponível em: <http://www.biotaneotropica.org.br/ v7n3/pt/abstract?article+bn03407032007>. Acessado em 15.11.2012.

Lucinda, P. H. F. 2003. Family Poeciliidae. In: ReIs, R. E.; Kullander, S. O. \& FerRaris JR., C. J. ed. Check list of the freshwater fishes of South and Central America. Porto Alegre, Edipucrs, p. 555-581.

Maistro, E. L.; Foresti, F. \& Oliveira, C. 1998. Comparative cytogenetic and morphological analysis of Astyanax scabripinnis paranae (Pisces, Characidae, Tetragonopterinae). Genetics and Molecular Biology 21(2):201-206.

Mariotto, S.; Moreira-Filho, O.; Bertollo, L. A. C.; Centofante, L. \& Mryazawa, C. S. 2009. Chromosome polymorphism in Ancistrus cuiabae Knaack, 1999 (Siluriformes: Loricariidae, Ancistrini). Neotropical Ichthyology 7:595-600.

Marques, D. K. S. \& Resende, E. K. 2005. Distribuição do tucunaré Cichla cf. monoculus (Osteichthyes, Cichlidae) no Pantanal. Boletim de Pesquisa e Desenvolvimento Embrapa-Pantanal 60:1-28.

Martins, F. O.; Marinho, M. M. F.; Langeani, F. \& Serra, J. P. 2012. A new species of Hypostomus (Siluriformes: Loricariidae) from the Upper Rio Paraguay Basin, Brazil. Copeia 2012(3):494-500.

Melo, F. A. G. 2005. Revisão taxonômica do complexo de espécies Astyanax fasciatus (Cuvier, 1819) (Teleostei: Characiformes: Characidae). Tese de Doutorado. Rio de Janeiro, Museu Nacional, UFRJ.

MMA. 1997. Plano de conservação da Bacia do Alto Paraguai (Pantanal): PCBAP. Análise integrada e prognóstico da bacia do Alto Paraguai. Brasília, Ministério do Meio Ambiente, dos Recursos Hídricos e da Amazônia Legal. $12 \mathrm{v}$.

Moreira, A. A.; Hilsdorf, A. W. S.; Silva, J. V. \& Souza, V. R. 2007. Variabilidade genética de duas variedades de tilápia nilótica por meio de marcadores microssatélites. Pesquisa Agropecuária Brasileira 42(4):521-526

Nelson, J. S. 2006. Fishes of the World. 4ed. Hoboken, John Wiley \& Sons. $611 \mathrm{p}$.

Oyakawa, O. T. \& Menezes, N. A. 2011. Checklist of fresh water fishes from São Paulo State, Brazil. Biota Neotropica 11(1a):1-13. Disponível em: <http://www.biotaneotropica.org.br/v11n1a/en/abstract?inventor

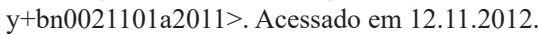

Reis, R. E.; Kullander, S. O. \& Ferraris. C. J. ed. 2003. Checklist of the freshwater fishes of South and Central America. Porto Alegre, EDIPUCRS. 729p. 
Retzer, M. E. \& Page, L. M. 1997. Systematic of the stick catfishes, Farlowella Eigenmann \& Eigenmann (Pisces, Loricariidae). Proceedings of the Academy of Natural Sciences of Philadelphia 147:33-88

Sabino, J. \& Prado, P. I. 2006. Vertebrados: síntese do conhecimento da diversidade biológica do Brasil. In: Lewinsohn, T. M. org. Avaliação do estado do conhecimento da diversidade brasileira. Brasília, Ministério do Meio Ambiente. vol. 2, p. 55-143.

Sabino, J.; Andrade. L.P. \& Bessa, E. 2012. Ecoturismo: valorizar a natureza para gerar negócios sustentáveis e renda. In: SABINO, J. org. Ecoturismo. Nas trilhas da biodiversidade brasileira. Campo Grande, Natureza em Foco, p. 13-21.

Súarez, Y. R.; Souza, M. M.; Ferreira, F. S.; Pereira, M. J.; Silva, E. A.; Ximenes, L. Q. L.; Azevedo, L. G.; Martins, O. C. \& Lima JR, S. E. 2011. Patterns of species richness and composition of fish assemblages in streams of the Ivinhema River basin, Upper Paraná River. Acta Limnologica Brasiliensia 23(2):177-188.

Weber, C. 2003. Subfamily Hypostominae (Armored catfishes). In: ReIS, R. E.; Kullander, S. O. \& Ferraris, C. J. eds. Check List of the Freshwater Fishes of South and Central America. Porto Alegre, Edipurcs, p. 351-372.

Zanatta, A. S.; Ramos, I. P.; Silva, R. J.; Langeani, F. \& Carvalho, E. D. 2010. Pisces, Siluriformes, Ictaluridae, Ictalurus punctatus (Rafinesque,
1818): First record in middle Paranapanema river reservoir, aquaculture and exotic species dispersion. Check List 6(4):589-591.

Zawadzky, C. H.; Tencatt, L. F. C. \& Froehlich, O. 2014. A new unicuspidteethed species of the genus Hypostomus Lacépède, 1803 (Siluriformes: Loricariidae) from the rio Paraguay basin. Neotropical Ichthyology 12(1):97-104.

Zawadzky, C. H.; Weber, C. \& Pavanelli, C. S., et al. 2008. Two new species of Hypostomus Lacépède (Teleostei: Loricariidae) from the upper rio Paraná basin, Central Brazil. Neotropical Ichthyology 6(3):403-412.

Zawadzky, C. H.; Weber, C. \& Pavanelli, C. S. 2010. A new dark-saddled species of Hypostomus (Siluriformes: Loricariidae) from the upper rio Paraguay basin. Neotropical Ichthyology 8(4):719-725.

Zawadzky, C. H.; Weber, C.; Pavanelli, C. S. \& Renesto, E. 2002. Morphological and biochemical comparison of two allopatrid populations of Hypostomus margaritifer (Regan, 1907) (Osteichthyes, Loricariidae) from the upper Paraná River basin, Brazil. Acta Scientiarum 24(2):499-505.

ZAWADZKI, C. H.; ReNESTO, E. \& BINI, L. M. 1999. Genetic and morphometric analysis of three species of the genus Hypostomus Lacépède, 1803 (Osteichthyes: Loricariidae) from the Rio Iguaçu basin. Revue Suisse de Zoologie 106(1):91-105.

Zoneamento Ecológico-Econômico. 2009. Zoneamento EcológicoEconômico do Mato Grosso do Sul. Disponível em: <http://www. semac.ms.gov.br/zeems/>. Acessado em 21.11.2012. 